\title{
Two New Polymorphs of the Organic Semiconductor 9,10-Diphenylanthracene: Raman and X-ray Analysis
}

Tommaso Salzillo ${ }^{1}$, Raffaele Guido Della Valle ${ }^{1}$, Elisabetta Venuti ${ }^{*}, 1$, Aldo Brillante ${ }^{1}$, Theo Siegrist ${ }^{2}$, Matteo Masino ${ }^{3}$, Francesco Mezzadri ${ }^{3}$, and Alberto Girlando ${ }^{*}$.

${ }^{1}$ Dip. di Chimica Industriale "Toso Montanari" and INSTM-UdR Bologna, Università di Bologna, Viale Risorgimento 4, I-40136 Bologna, Italy.

${ }^{2}$ Dept. of Chemical and Biomedical Engineering, FAMU-FSU College of Engineering, Florida State University and Florida A\&M University, Tallahassee, FL 32310-6046, U.S.A..

${ }^{3}$ Dip. di Chimica and INSTM-UdR Parma, Università degli Studi di Parma, Area delle Scienze 17/A I-43124 Parma, Italy.

E-mail: elisabetta.venuti@unibo.it; alberto.girlando@unipr.it

\section{Supporting Information}

\section{SI content}

1. Raman bands in the $0-150 \mathrm{~cm}^{-1}$ energy range............................pag. S2

2. Powder XRD....................................................................... S2

3. Computed free energy $G(p, T)$ vs temperature $T$ (at ambient $p$ )...........pag. S3

4. HOMO and LUMO hopping integrals for the DPA polymorphs...........pag. S4 


\section{Raman bands in the $0-150 \mathrm{~cm}^{-1}$ energy range}

Table S 1. Wavenumber of the bands observed in the Raman spectra of the three DPA polymorphs in the energy range $0-150 \mathrm{~cm}^{-1}$.

\begin{tabular}{|c|c|c|c|c|c|}
\multicolumn{2}{c|}{$\boldsymbol{\alpha - D P A}(\mathbf{C} 2 / \mathbf{c})$} & \multicolumn{2}{c|}{$\boldsymbol{\beta}$-DPA (P2 1 /a) } & \multicolumn{2}{c|}{$\boldsymbol{\gamma}$-DPA (P2 1 /n) } \\
\hline \hline$\omega\left(\mathrm{cm}^{-1}\right)$ & Pol. & $\omega\left(\mathrm{cm}^{-1}\right)$ & Pol. & $\omega\left(\mathrm{cm}^{-1}\right)$ & Pol. \\
\hline 45 & $(y, y)(x, y)$ & 33 & $(y, y)(x, y)$ & 22 & $(x, x)(y, y)(x, y)$ \\
\hline 55 & $(x, x)(y, y)(x, y)$ & 40 & $(x, x)(y, y)(x, y)$ & 27 & $(x, x)(y, y)(x, y)$ \\
\hline 60 & $(x, x)(y, y)(x, y)$ & 44 & $(y, y)(x, y)$ & 41 & $(x, x)(x, y)$ \\
\hline 69 & $(x, x)(x, y)$ & 50 & $(x, x)(y, y)(x, y)$ & 45 & $(x, x)(x, y)$ \\
\hline 76 & $(y, y)(x, y)$ & 59 & $(y, y)(x, y)$ & 50 & $(x, x)(x, y)$ \\
\hline 95 & $(y, y)(x, y)$ & 75 & $(y, y)(x, y)$ & 61 & $(x, x)(x, y)$ \\
\hline 99 & $(x, x)(y, y)(x, y)$ & 92 & $(x, x)(x, y)$ & 78 & $(x, x)(y, y)(x, y)$ \\
\hline 110 & $(x, x)(y, y)(x, y)$ & 99 & $(y, y)(x, y)$ & 89 & $(x, x)(y, y)(x, y)$ \\
\hline 118 & $(x, x)(x, y)$ & 115 & $(y, y)(x, y)$ & 105 & $(x, x)(y, y)(x, y)$ \\
\hline & & & & 116 & $(x, x)$ \\
\hline
\end{tabular}

\section{Powder XRD}

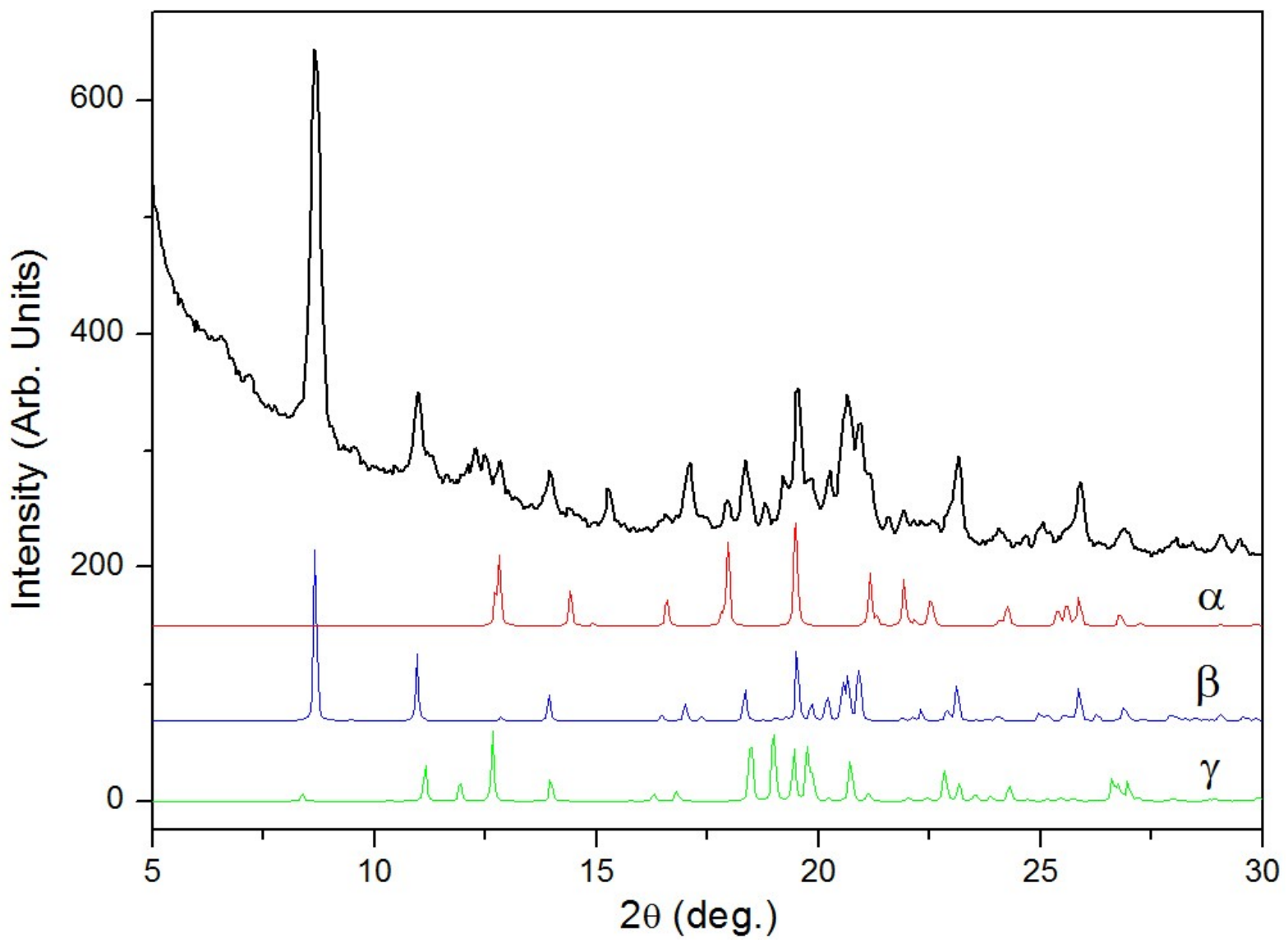

Figure S1. Powder X-ray diffraction of the micro-crystals compared with the simulated powder diffraction of the three polymorphs of DPA. 
3. Computed free energy $G(p, T)$ vs temperature $T$ (at ambient $p)$.

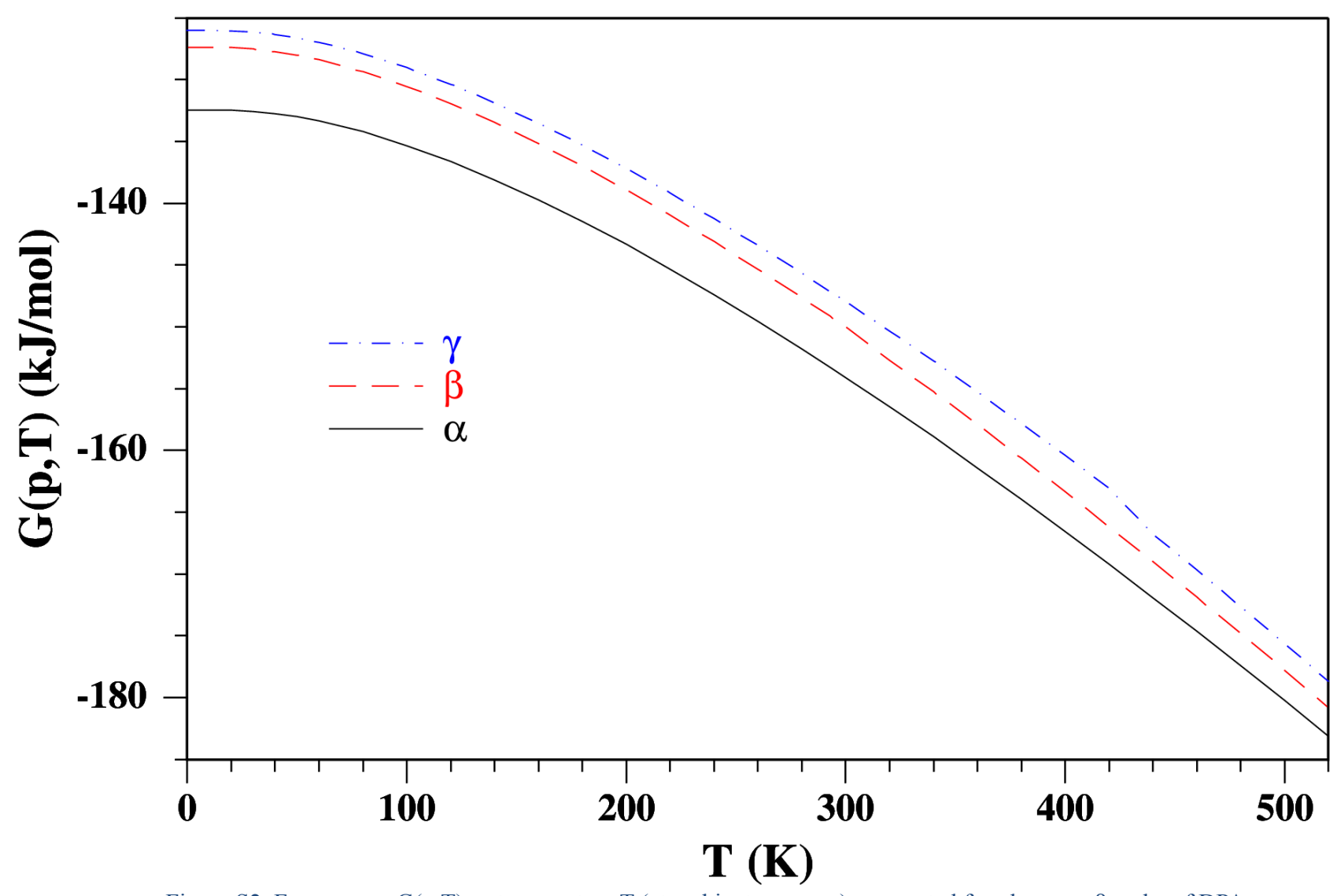

Figure S2. Free energy $\mathrm{G}(\mathrm{p}, \mathrm{T})$ vs temperature T (at ambient pressure), computed for phases $\alpha, \beta$ and $\gamma$ of DPA. 
4. HOMO and LUMO hopping integrals for the DPA polymorphs

Table S2. Hopping integrals (meV) of the three DPA polymorphs, of Rubrene and of Anthracene

\begin{tabular}{|l|c|c|c|c|c|}
\hline & $\begin{array}{c}\text { DPA } \\
\text { HOMO, LUMO }\end{array}$ & $\begin{array}{c}\text { DPA } \\
\text { HOMO, LUMO }\end{array}$ & $\begin{array}{c}\text { DPA } \\
\text { HOMO, LUMO }\end{array}$ & $\begin{array}{c}\text { Rubrene } \\
\text { HOMO, LUMO }\end{array}$ & $\begin{array}{c}\text { Anthracene } \\
\text { HOMO, } \\
\text { LUMO }\end{array}$ \\
\hline $\boldsymbol{t}_{\mathbf{1}}$ & 20,5 & 33,13 & 18,14 & 84,39 & 27,38 \\
\hline $\boldsymbol{t}_{\mathbf{2}}$ & 4,2 & 4,0 & 1,12 & 10,4 & \\
\hline $\boldsymbol{t}_{\mathbf{3}}$ & 29,5 & 0,0 & & & \\
\hline $\boldsymbol{t}_{\mathbf{4}}$ & 0,3 & & & & \\
\hline $\boldsymbol{t}_{\mathbf{5}}$ & 5,2 & & & & \\
\hline
\end{tabular}

